\title{
- Galactic Debris as a Dark Matter Identifier
}

\author{
Dynamic maps of 12 stellar streams trace paths that are determined by the \\ distribution of mass in the Milky Way.
}

By Rachel Berkowitz

$1 /$

hen our Galaxy ensnares a neighboring globular cluster, the debris from that merger stretches into an arc called a stellar stream. Researchers are interested in measuring these streams, as it is hypothesized that their paths could be deflected by nearby dark matter. But observing stellar streams is difficult because their member stars are sparsely distributed against the bright foreground of the Milky Way's disk. Now, Ting Li from the University of Toronto, Canada, and her colleagues have successfully mapped the orbits, velocities, and chemical compositions of 12 stellar streams within our Galactic halo, the largest number of streams that have been mapped simultaneously. The maps could provide new information about the mass budget of the Milky Way, the distribution of dark matter within our Galaxy, and the properties of dark matter itself.

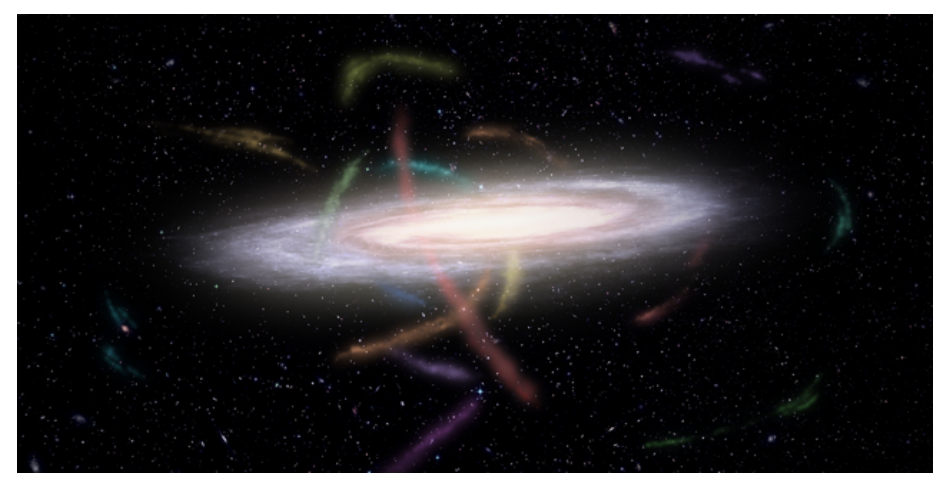

Artistic representation of the Milky Way surrounded by dozens of stellar streams-satellite galaxies or globular clusters that are being pulled apart by our Galaxy's gravity.

Credit: J. Josephides/S5 Collaboration
Stellar streams are the remnants of ancient mergers of our Galaxy with other galaxies and with other stellar structures and thus provide insight into the history of our Galaxy. They have also been touted as the missing puzzle piece that could explain the Milky Way's structure (see Mapping Dark Matter in the Milky Way), as their paths are governed by the distribution of mass in the Galaxy.

Observations from stellar streams could therefore provide clues for determining the makeup of the Milky Way's visible and invisible matter, which, in turn, could help in evaluating dark matter models. For example, some models predict that dark matter comes together in "clumps" in both the Milky Way and in other galaxies. There could also be "dark satellite galaxies" that we cannot see directly but that could be mapped with stellar streams, Li says.

To measure a stellar stream's properties, Li and her colleagues at the Southern Stellar Stream Spectroscopic Survey $\left(S^{5}\right)$ use the Anglo-Australian Telescope to determine the line-of-sight velocities of individual stars within the stream. Those observations rely on the Doppler shift of light to pinpoint a star and to determine how fast it is moving. But to conclusively find a stellar-stream star this way, the team has to examine around 100 stars.

Crucial to the success of the $S^{5}$ Collaboration's maps are data from the Gaia space mission. Compared to data from ground-based measurements, those made by the Gaia space observatory can be used to more quickly and efficiently determine whether a target star is part of a stream, because its observations are not blurred by gases in Earth's atmosphere, $\mathrm{Li}$ 
says. "Instead of 100 , we only need to examine 10 [stars]" to identify one in a stream. Gaia can also be used to measure a star's composition and its speed in the plane of the sky, providing chemistry information for each stream and 3D velocity information for each stream member.

Working backward from those observations, the team used computer simulations to ascertain where and from what each stream originated. For example, they identified some streams emanating from the remains of globular clusters and others from disrupted dwarf galaxies. They also found that the streams were chemically distinct from globular clusters and dwarf galaxies that are still intact. Vasily Belokurov, an astronomer at the University of Cambridge, UK, notes that many of the observed streams associated with globular clusters have more hydrogen and helium (lower "metallicity") relative to intact globular clusters. "This new study is the first to systematically measure and analyze a large number of stellar streams," Belokurov says.

Li and her colleagues plan to make measurements of other Milky Way stellar streams. Meanwhile, they say that they are currently working on analyzing the data they have collected thus far for information about the mass distribution and properties of the Milky Way's dark matter. "This dataset is clearly a treasure trove for global studies of the Milky Way density field," Belokurov says.

Rachel Berkowitz is a Corresponding Editor for Physics based in Vancouver, Canada. 